\title{
Diagnostic Importance of Flair and Diffusion Weighted Magnetic Resonance Imaging of the Atypic Intracranial Epidermoid Cysts
}

\author{
Duygu Kara Bozkurt, Mustafa Gök, Ahmet Erdem, Adem Kiriş \\ Department of Radiology, Kafkas University Faculty of Medicine, Kars, Turkey
}

\begin{abstract}
Epidermoid cysts (ECs) are benign, slow-growing, congenital tumors. Intracranial ECs usually localize in extra-axial spaces, particularly in the cerebellopontine angle or in the parasellar region. Their occurrences in the fourth ventricle or intradiploic location are exceptionally rare. We present the imaging findings in two patients with atypical localization of ECs. Magnetic resonance imaging (MRI) findings for the first case revealed an intraventricular EC, and the second case demonstrated an EC that invaded into the intradiploic spaces and the calvarium. Conventional MRI, including T1 and $\mathrm{T} 2$ weighted imaging $(\mathrm{T} 1 \mathrm{Wl}$ and $\mathrm{T} 2 \mathrm{WI})$, may not always differentiate ECs from other cystic intracranial lesions. In addition to conventional MRI findings, diffusion-weighted imaging (DWI) and fluid attenuation invention recovery (FLAIR) sequences play an important role in the differentiation of ECs from other intracranial cystic lesions, thereby reflecting the contents of the lesions. In conclusion, we recommend the use of FLAIR and DWI sequences in addition to conventional MRI sequences for the differential diagnosis of the atypical localized epidermoid tumors. (JAREM 2015; 5: 134-8)
\end{abstract}

Keywords: Magnetic resonance imaging, epidermoid cyst, fourth ventricle, intradiploic space

\section{INTRODUCTION}

Epidermoid cysts (ECs) are benign, slow-growing, congenital lesions arising from epithelial remnants during the neural tube closure between the $3^{\text {rd }}$ and $5^{\text {th }}$ week of gestation (1). Pathologically, the wall of the tumor comprises keratinizing squamous epithelium and fibrous connective tissue. The cyst is filled with cholesterol crystals, keratin, protein, debris, and cerebrospinal fluid (CSF) (2). ECs commonly occur in the cerebellopontine angles, suprasellar, and parasellar regions. In contrast, ECs in the fourth ventricle or intradiploic locations are very rare, accounting for less than $5 \%$ of all intracranial ECs (3). Because of the locations, fourth ventricle ECs may cause a mass effect on brainstem structures; intradiploic space ECs may invade the calvarium, meninges, and adjacent brain parenchyma. The margins of the tumor should be revealed for appropriate surgical management. In these two rare cases, we discuss the diagnostic importance of fluid attenuation invention recovery (FLAIR) and diffusion-weighted imaging (DWI) for the atypical localized ECs.

\section{CASE PRESENTATIONS}

\section{CASE 1}

A 47-year-old female was admitted to our hospital with a history of long-standing headache. In the last 2 years, the patient complained of dizziness during walking and did not undertake any activities such as sports or dancing. Upon physical examination, there were no neurological deficits. Laboratory findings were within normal limits. Magnetic resonance imaging (MRI) was performed on a 1.5-Tesla clinical MR system (Siemens Medical System, Magnetom Essenza, Germany). Conventional MRI findings (includes T1WI and T2WI) showed T1WI hypointense and T2WI hyperintense cystic lesions with sizes of $50 \times 33 \mathrm{~mm}$ located in the fourth ventricle, which protruded toward the foramen Luschka and extended to the left cerebellar hemisphere
(Figures 1a, b). The lesion shifted the medulla oblongata to the right side. Despite its size and mass effect on the brain stem, no significant vasogenic edema was evident. On conventional MRI, the lesion appeared isointense to CSF and was not enhanced after contrast infusion. On FLAIR imaging, the lesion appeared heterogeneously hyperintense relative to CSF (Figure 1c). Unlike conventional MRI, FLAIR imaging indicated that the nature of the lesion was not purely cystic. Because of the hyperintensity on FLAIR imaging, we decided to perform DWI to understand if the lesion is solid or cystic. Echo-planar DWI and apparent diffusion coefficient (ADC) mapping were performed, and ADC values (the main quantitative parameter obtained from DWI) were calculated on a computer workstation. On echo-planar DWI sequences, the lesion was highly hyperintense relative to CSF and adjacent cerebral parenchyma (Figure 1d), whereas the lesion appeared distinctly hypointense relative to CSF and adjacent cerebral parenchyma on ADC mapping (Figure 1e). The mean ADC value of the lesion was $1127 \times 10^{-6} \mathrm{~mm}^{2} / \mathrm{s}$, adjacent cerebral parenchyma was $915 \times 10^{-6} \mathrm{~mm}^{2} / \mathrm{s}$, and CSF was $3150 \times 10^{-6} \mathrm{~mm}^{2} / \mathrm{s}$. The range of the lesion ADC values were observed between CSF and cerebral parenchyma values. These quantitative values explained that the content of the lesion was not purely cystic. All radiological findings were suggestive of EC as a diagnosis. Intraoperative findings and histological examination of the resected lesion revealed a typical stratified squamous epithelium containing keratinaceous debris findings consistent with the findings for EC. The patient recovered well following surgery and had no neurological sequelae. A follow-up MRI after 6 months revealed no residual tumor or evidence of tumor recurrence. The patient provided her informed consent for the publishing of data.

\section{CASE 2}

A 74-year-old female patient was referred to our hospital with a 1 -year history of left proptosis with painless swelling of the up- 
per and lateral bulbar conjunctiva. In the last 6 months, the patient complained of limited left eye movement, particularly lateral movement. Laboratory findings were within normal limits. Computed tomography (CT) (Aquilion 64; Toshiba, Japan) showed the destruction of the left temporal bone and sphenoid wing by an extracerebral, hypodense, intradiploic tumor. In bone window, the CT scan revealed erosions in both inner and outer table of the calvarium and compression on the left temporal lobe and left lateral rectus muscle (Figures 2a, b). MRI was performed on a 1.5-Tesla clinical MR system (Magnetom Essenza 1.5T; Siemens, Erlangen, Germany). On conventional MRI examination, the mass was demonstrated to be homogenously hypointense on T1WI and hyperintense on T2WI with a size of $26 \times 18 \mathrm{~mm}$ (Figures $2 \mathrm{c}$, d). The lesion was not enhanced after contrast administration. On conventional MRI findings, the content of the lesion was purely cystic, but FLAIR imaging showed heterogenously hyperintense (compared with CSF) lesion (Figure 2e). This indicated that the nature of the lesion was not purely cystic. Because of the hyperintensity on FLAIR imaging, we decided to perform DWI to explain solid or cystic nature of the lesion. Echo-planar DWI and ADC mapping were performed and $A D C$ values (the main quantitative parameter obtained from DWI) were calculated. On echo-planar DWI sequences, the lesion showed diffusion restriction compatible with highly hyperintense relative to CSF and adjacent cerebral parenchyma (Figure 2f), whereas the lesion appeared distinctly hypointense relative to CSF and adjacent cerebral parenchyma on ADC mapping (Figure $2 \mathrm{~g}$ ). The mean ADC value of the lesion was $1072 \times 10^{-6} \mathrm{~mm}^{2} / \mathrm{s}$, adjacent cerebral parenchyma was $895 \times 10^{-6} \mathrm{~mm}^{2} / \mathrm{s}$, and CSF was $2850 \times$ $10^{-6} \mathrm{~mm}^{2} / \mathrm{s}$. The ADC value of the lesion was between CSF and cerebral parenchyma. These quantitative values explained that the content of the lesion was not purely cystic. All radiological findings were suggestive of EC as a diagnosis. Intraoperative findings and histological examination of the resected lesion revealed typical stratified squamous epithelium containing keratinaceous debris findings consistent with the findings for an EC. The patient recovered well following surgery and had no neurological sequelae. A follow-up MRI after 6 months revealed no residual tumor or evidence of tumor recurrence. The patient provided her informed consent for the publishing of data.

\section{DISCUSSION}

Intracranial ECs are benign, congenital tumors that arise from displaced epithelial remnants that remain after the neural tube closure, accounting for approximately $1 \%$ of the intracranial tumors (4). The most common intracranial locations of ECs are cerebellopontine angle, parasellar region, and suprasellar cistern. They may occur less frequently in the lateral or fourth ventricle, intradiploic space, cerebellum, and brainstem $(5,6)$. In the literature, few cases of ECs located in the fourth ventricle and intradiploic space have been reported because of the low incidence of ECs. ECs generally remain asymptomatic for long periods of development; however, they become symptomatic in the later periods secondary to expansile overgrowth or rupture.

In some cases, expansile overgrowth may cause rupture, and the leakage of cyst contents produces chemical irritation of the leptomeninges or ependyma (7). Most of the symptoms mainly depend on the location and the mass effect of the lesion rather than the natural course of the disease (8).
Our first patient presented with a history of long-standing headache, which could be attributable to the local effects of the tumor. The local effects of the fourth ventricle ECs may cause obstructive symptoms such as increased intracranial pressure because of the blockage of the CSF flow. The other important symptom is related to the mass effect on the brainstem. Similar to our patient, these patients complain of long lasting headaches, vertigo, dizziness, and mental disorders $(1,9)$. Our second patient complained about the limitation of the left eye movements, which could be related to the local effects of the tumor. Intradiploic ECs may erode the inner and the outer layer of the calvarium causing a mass effect on the adjacent structure. In the second patient, a tumor in the intradiploic space of the sphenoid wing may cause compression on the lateral rectus muscle to restrict the eye movements. In both cases, all the symptoms disappeared after total surgical resection of the lesions. In the pre-operative period, determining the localization and nature of the lesion is important in improving the success rate of surgery. Therefore, in combination with conventional MRI, FLAIR and DWI imaging provide additional information about the localization and nature of the lesion.

In general, the typical MRI appearance of cystic lesions is hypointense on T1WI and hyperintense on T2WI, which is similar to CSF intensity. On conventional MRI (T1WI and T2WI), typical epidermoid and arachnoid cysts share similar T1 and T2 signals and is not enhanced after contrast administration; thus, the differentiation between them requires FLAIR and DW imaging. Also, epidermoid tumors usually exhibit poor contrast from surrounding $\mathrm{CSF}$, and it is difficult to reveal the contour of the lesion on conventional MRI (10).

MR signals depend on the cholesterol and keratin contents of EC; generally, the cholesterol in an epidermoid is in a solid state and appears hypointense on T1WI $(1,2)$. In some cases, atypic ECs seem to be iso- or hyperintense on T1WI because of their high protein content; on $\mathrm{T} 2 \mathrm{Wl}$, the signal intensity decreases with increasing protein concentration (2).

Conventional MRI plays an important role in planning of a surgical approach and FLAIR imaging provides additional information about the lesion by suppressing the signal of the surrounding CSF and intensifying the long-T2 quality of the lesions. In our cases, all epidermoid tumors showed significantly hyperintense signals relative to CSF from which the lesions certainly could be discriminated, although they also showed unclear tumor margins. FLAIR imaging does not show ECs as hyperintense as in T2WI. Instead, they are seen in intermediate intensity between brain parenchyma and CSF; with this extra information, we are having suspicion about the nature of the lesions (11). In our cases, epidermoid tumors are minimally hyperintense relative to the brain parenchyma in FLAIR imaging.

DWI based on the random diffusion (Brownian motion) of water molecules in tissues is increasingly being used to investigate intracranial pathologies. The main parameter obtained from DWI is the ADC. The main factors affecting the ADC values are tissue architecture, molecular interactions, and cellular density. Thus, $A D C$ is altered by various physiological and pathological conditions of organ systems (11). 

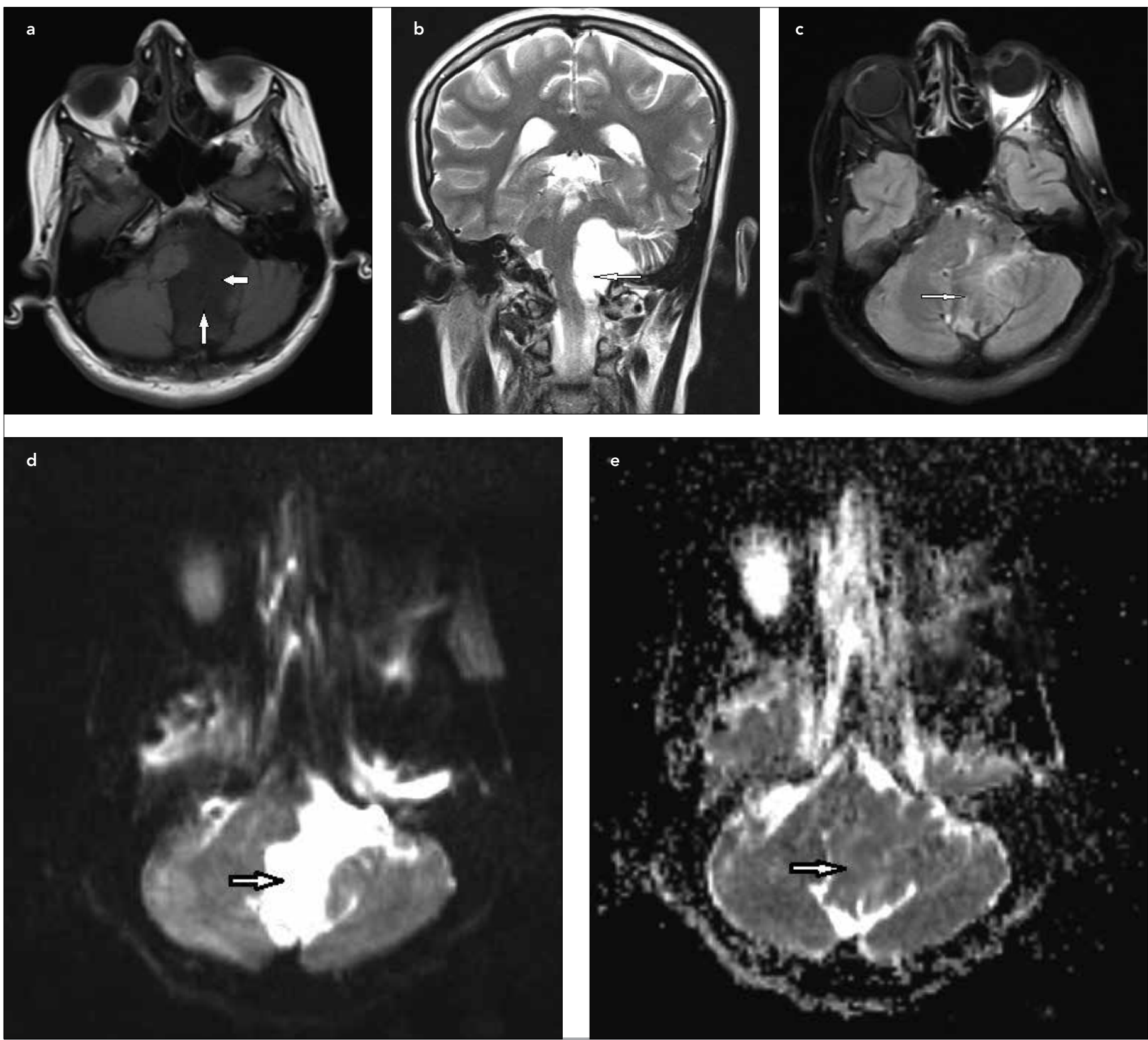

Figure 1. a-e. A 47-year-old woman with EC of the fourth ventricle. Axial T1WI of the brain shows hypointense cystic lesion located in the fourth ventricle protruding toward the foramen Luschka and extends to the left cerebellar hemisphere (white arrows) (a). Coronal T2WI shows hyperintense cystic lesion (arrow) with a shifted medulla oblongata to the right side (b). Axial FLAIR images show heterogenously hyperintense lesion relative to CSF with unclear tumor margins (arrow) (c). DWI with $b=1,000 \mathrm{~mm}^{2} / \mathrm{s}$ clearly shows a well-defined hyperintense mass in the fourth ventricle protruding towards the foramen Luschka and extends to the left cerebellar hemisphere (d). On the ADC map, the tumor is hypointense (arrow). The ADC value within the mass is $1127 \times 10^{-6} \mathrm{~mm}^{2} / \mathrm{s}(\mathrm{e})$

The low ADC values of ECs result from the dense keratinous and proteinaceous content of the cyst, which limits the motion of the free protons causing hyperintensity on DWls (12). The hyperintensity of ECs in DWI is affected by ADC values as well as T2 values and proton density. It is reported that the ADCs of epidermoid tumors were similar to those of brain tissue, whereas the ADCs of cystic lesions were similar to those of CSF (13).

In our cases, all epidermoid tumors appeared sharply hyperintense relative to the brain and CSF. The fact that the mean ADC of epidermoid tumors was $1127 \times 10^{-6} \mathrm{~mm}^{2} / \mathrm{s}$ and $1072 \times 10^{-6}$ $\mathrm{mm}^{2} / \mathrm{s}$, respectively, higher than the brain parenchyma, implies more active diffusion in the epidermoid tumors than in the brain parenchyma. In the literature, DWI sequences greatly aided in the evaluation of the postoperative patients. On conventional MRI, postoperative changes in the surgical cavity usually make the detection of residual tumor or recurrence more difficult (9). In our cases, post-surgical follow-up MRI studies, including DW and FLAIR imaging, showed no residual tumor or recurrence.

Although ECs involving the fourth ventricle are extremely rare, they should be considered in the differential diagnosis of the 

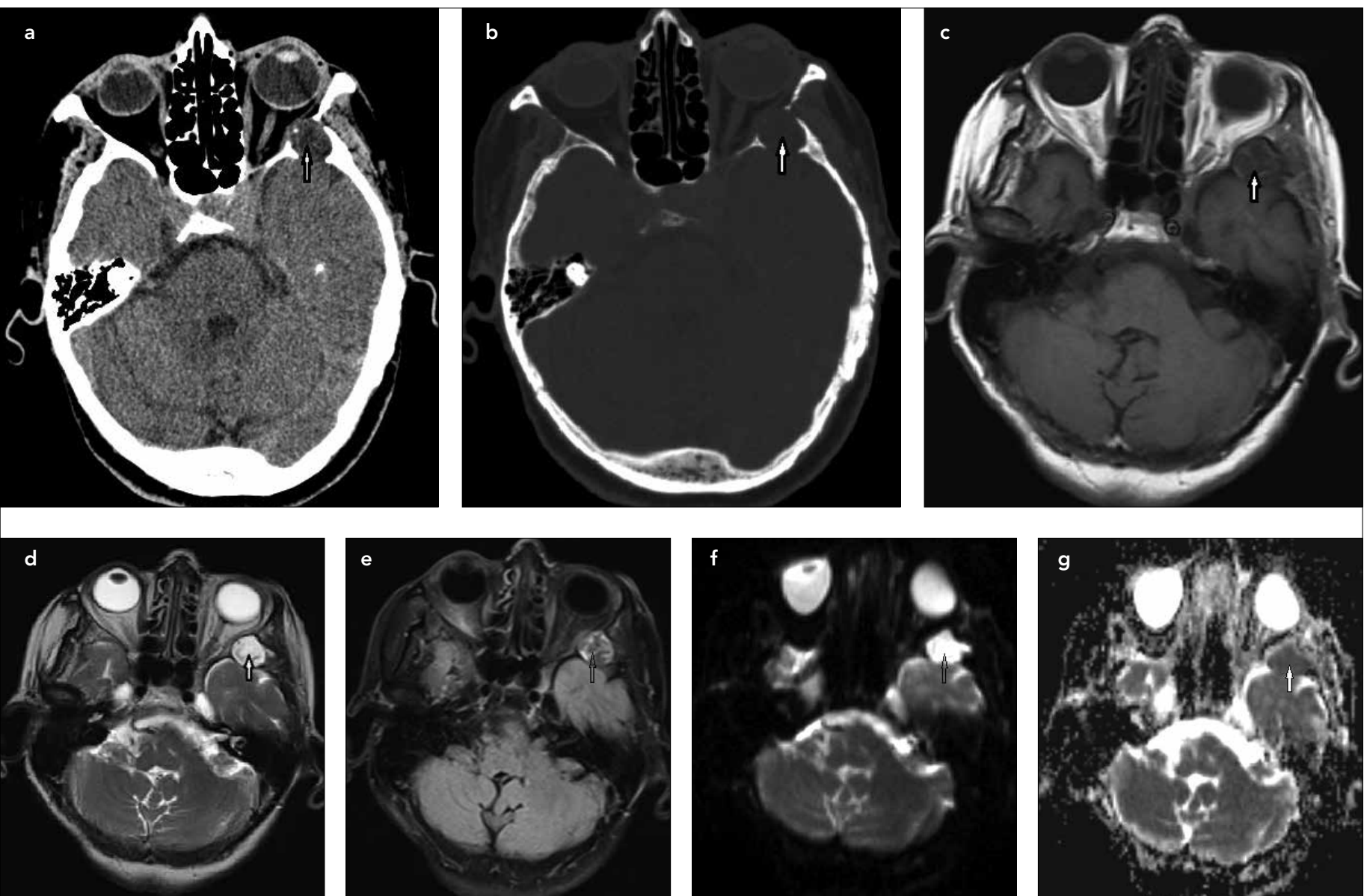

Figure 2. a-g. A 74-year-old woman with $E C$ in the intradiploic space of the sphenoid wing. Axial non-enhanced CT image of the brain shows a hypointense lesion (arrow) with regular contour in the diploic space of the sphenoid wing that invades the left temporal lobe and the lateral rectus muscle (a). Axial non-enhanced CT image in bone windows shows erosion of both inner and outer table of the calvarium (b). On axial T1WI, the lesion is homogenously hypointense (arrow) (c). On axial T2WI, the lesion is homogenously hyperintense (arrow) (d). Axial FLAIR images shows heterogenously hyperintense lesion relative to CSF (arrow) (e). DWI with $b=1,000 \mathrm{~mm}^{2} / \mathrm{s}$ clearly shows a well-defined hyperintensity mass in the sphenoid wing (f). On the ADC map, the lesion is hypointense (arrow). The ADC value within the mass is $1072 \times 10^{-6} \mathrm{~mm}^{2} / \mathrm{s}(\mathrm{g})$

intraventricular tumor, particularly in the fourth ventricle. The differential diagnosis includes the following: arachnoid cyst, lipomatous hamartoma, dermoid cyst, and cystic neoplasms (14). Intradiploic epidermoids are to be differentiated from dermoid cysts, eosinophilic granulomas, cholesterol granulomas, and hemangiomas on CT and MR images. Aneurysmal bone cysts, fibrous dysplasia, and eosinophilic granuloma are also included in the differential diagnosis of expansile lytic lesions in young adults and children (15). Radiologically, dermoid cyst contents usually resemble fat on CT and MRI, whereas the contents of an epidermoid cyst resemble CSF similar to our patients.

In treatment options, total surgical excision is recommended. The dense attachment to surrounding structures is one of the major problem to surgical treatment. Extensive lesions, incomplete initial resection, and limited initial exposure are considered as the primary causes of recurrence. Even with advance surgical techniques, the total surgical resection rates are not more than $30 \%(16)$.

\section{CONCLUSION}

Differentiation of ECs from other intracranial cystic lesions is important for an appropriate surgical management. When added to conventional MRI findings, DW and FLAIR imaging become useful functional imaging tools for the epidermoid tumors and give more information regarding the nature and invasion of the lesions and for monitoring the therapeutic outcome after surgical resection.

Informed Consent: Written informed consent was obtained from the patients who participated in this case.

Peer-review: Externally peer-reviewed.

Author Contributions: Concept - D.K.B., M.G.; Design - D.K.B.; Supervision - D.K.B., M.G., A.K.; Resources - D.K.B., M.G., A.E.; Data Collection and/or Processing- D.K.B., A.E.; Analysis and/or Interpretation - D.K.B., M.G., A.K.; Literature Search - D.K.B., M.G.; Writing Manuscript - D.K.B.

Conflict of Interest: No conflict of interest was declared by the authors.

Financial Disclosure: The authors declared that this study has received no financial support.

\section{REFERENCES}

1. Mermuys K, Wilms G, Demaerel P. Epidermoid cyst of the fourth ventricle: diffusion-weighted and flair MR imaging findings. JBR-BTR 2008; 9: 58-60. 
2. Ochi M, Hayashi K, Hayashi T, Morikawa M, Ogino A, Hashmi R, et al. Unusual CT and MR appearance of an epidermoid tumor of the cerebellopontine angle. AJNR Am J Neuroradiol 1998; 19: 1113-5.

3. Aribandi M, Wilson NJ. CT and MR imaging features of intracerebral epidermoid-a rare lesion. Br J Radiol 2008; 81: e97-9. [CrossRef]

4. Hsieh $\mathrm{CH}$, Huang KM, Kao MC, Peng S, Wang CC. Hemorrhage in intracranial epidermoid cyst. J Formos Med Assoc 1996; 95: 173-5.

5. Koot RW, Jagtap AP, Akkerman EM, Den Heeten GJ, Majoie CB. Epidermoid of the lateral ventricle: evaluation with diffusionweighted and diffusion tensor imaging. Clin Neurol Neurosurg 2003; 105: 270-3. [CrossRef]

6. Jeon JY, Kim JM, Cheong JH, Kim CH. Epidermoid Cyst of the Fourth Ventricle. J Korean Neurosurg Soc 2005; 38: 478-80.

7. Guttal KS, Naikmasur VG, Joshi SK, Bathi RJ. Trigeminal neuralgia secondary to epidermoid cysts at the cerebellopontine angle: case report and brief overview. Odontology 2009; 97: 54-6. [CrossRef]

8. Ulrich J. Intracranial Epidermoids. A study on their distribution and spread. J Neurosurg 1964; 21: 1051-8. [CrossRef]

9. Tancredi A, Fiume D, Gazzeri G. Epidermoid cysts of the fourth ventricle: very long follow up in 9 cases and review of the literature. Acta Neurochir (Wien) 2003; 145: 905-10. [CrossRef]
10. Hakyemez B, Aksoy U, Yildiz H, Ergin N. Intracranial epidermoid cysts: diffusion-weighted, FLAIR and conventional MR findings. Eur J Radiol 2005; 54: 214-20. [CrossRef]

11. Chen S, Ikawa F, Kurisu K, Arita K, Takaba J, Kanou Y. Quantative MR evaluation of intracranial epidermoid tumors by fast fluid-attenuated inversion recovery imaging and echo-planar diffusion-weighted imaging. AJNR Am J Neuroradiol 2001; 22: 1089-96.

12. Laing AD, Mitchell PJ, Wallace D. Diffusion-weighted magnetic resonance imaging of intracranial epidermoid tumours. Australas Radiol 1999; 43: 16-29. [CrossRef]

13. Tsuruda JS, Chew WM, Moseley ME, Norman D. Diffusionweighted MR imaging of the brain: value of differentiating between extraaxial cysts and epidermoidtumors. AJR Am J Roentgenol 1990; 155: 105965. [CrossRef]

14. Osborn AG, Preece MT. Intracranial cysts: radiologic-pathologic correlation and imaging approach. Radiology 2006; 239: 650-64. [CrossRef]

15. Arana E, Martí-Bonmatí L. CT and MR imaging of focal calvarial lesions. AJR Am J Roentgenol 1999; 172: 1683-8. [CrossRef]

16. Murakami N, Matsushima T, Kuba H, Ikezaki K, Morioka T, Mihara F, et al. Combining steady-state constructive interference and diffusionweighted magnetic resonance imaging in the surgical treatment of epidermoid tumors. Neurosurg Rev 1999; 22: 159-62. [CrossRef] 